\title{
ESPAÑA Y LA ORGANIZACIÓN MUNDIAL DE LA SALUD EN TIEMPOS DE PALANCA: UNA EVALUACIÓN PROVISIONAL
}

\author{
Esteban Rodríguez-Ocaña \\ Dpto Historia de la Ciencia \\ Universidad de Granada \\ erodrig@ugr.es \\ ORCID iD: https://orcid.org/0000-0003-4195-4487
}

Recibido: 26 junio 2018; Aprobado: 5 marzo 2019.

Cómo citar este artículo/Citation: Rodríguez-Ocaña, Esteban (2019), “España y la Organización Mundial de la Salud en tiempos de Palanca: una evaluación provisional", Asclepio, 71(1): p254. https://doi.org/10.3989/asclepio.2019.06

RESUMEN: España ingresó en la Organización Mundial de la Salud en 1952, estando el General José Alberto Palanca al frente de la Dirección General de Sanidad y hasta 1957. Con él se iniciaron diversos proyectos de colaboración con la misma. Empleando como principal fuente documentación procedente de los archivos de la OMS, me planteo cómo se desarrollaron dichas relaciones y cuáles fueron sus efectos sobre las dinámicas científica y profesional hispanas durante ese primer sexenio, teniendo en cuenta que el objetivo primario de la incorporación española fue de orden político. Ello no obstante, la colaboración con la OMS era conveniente para integrarse en las redes internacionales de producción e intercambio científico-médico, aunque en los primeros seis años sus efectos internos fueron escasos.

PALABRAS-CLAVE: Relaciones internacionales, administración sanitaria, España, franquismo.

\author{
SPAIN AND THE WORLD HEALTH ORGANIZATION \\ IN TIMES OF PALANCA: A FIRST ASSESSMENT
}

\begin{abstract}
Spain joined the World Health Organization in 1952, at the time of General José Alberto Palanca as head of the Spanish health administration, which lasted until 1957. Under his office several collaborative projects were started. Using as main source material from the Archives of WHO, I study how such relationships were performed and developed. Notwithstanding the fact that the WHO partnership was sought by the Spanish government as a political strategy, evidence suggests that collaboration with the WHO was a convenient step to get Spain into the international scientific medical network of production and exchange of knowledge, tools and practices, even though in the first six years this had little effects on the Spanish society.
\end{abstract}

KEY WORDS: International relations, Health administration, Spain, Francoism.

Copyright: @ 2019 CSIC. Este es un artículo de acceso abierto distribuido bajo los términos de la licencia de uso y distribución Creative Commons Reconocimiento 4.0 Internacional (CC BY 4.0). 
La conciencia contemporánea acerca de la dimensión internacional de la salud, que ha tomado cuerpo en el concepto de salud global, nos exige que en la reconstrucción de las políticas científicas y sanitarias del franquismo haya que tomar en cuenta el peso que dichas relaciones tuvieron en la situación de España, algo que estaba ausente de la mas reciente monografía (Rodríguez-Ocaña y Martínez Navarro, 2008). El análisis de las agencias internacionales con anterioridad a la II Guerra Mundial registra en los últimos años un destacado interés historiográfico (Iriye, 2002; Cueto, 2004; Solomon, Murard y Zylberman 2008; Borowy, 2009 y 2014), donde abundan los trabajos dedicados a la dimensión trasnacional sanitaria de la Fundación Rockefeller, su influencia en las políticas regionales y locales y su significado histórico (Stapleton, 2000; Rodríguez-Ocaña, 2000, 2002 y 2014; Löwy, Zylberman, 2000; Farley, 2004; Birn, 2006, 2014a y 2014b; Barona, 2015). La historia de la OMS fue, desde sus inicios, un foco de interés prioritario para la propia institución que ha ido publicando una serie informal recopilatoria de las actividades desarrolladas por decenios (OMS, 1958; OMS, 1968; Litsios, 2009 y 2012) o bien con motivo del cincuentenario de la misma (Hussein, 1998a; Hussein, 1998b). Este criterio cronológico responde también a los cambios en orientaciones, contenidos y programas del organismo internacional por lo que se ha mantenido en la historiografía (Roemer, 1993; Brown, Cueto y Fee, 2006; Brown, Cueto, 2011; Cueto, Brown, Fee, 2011). No existe en la bibliografía mundial, hasta donde hemos encontrado, ningún estudio sistemático sobre la relación de un determinado país miembro (no colonizado) con la OMS.

¿Qué implicaciones tiene la pertenencia a dicha organización internacional? La OMS ha sido considerada de forma genérica como una mas de las organizaciones tecnocráticas descritas por politólogos y sociólogos (Barnett y Finnemore 1999), a la vez que como una institución basada en la producción y diseminación de conocimiento, muy relacionada con el concepto de "comunidad epistémica" desarrollado por la sociología del conocimiento (Haas, 1992). Como tal, genera normas y estándares científicos y, a la vez, crea saber social, pues las normas incorporan, representan y dirigen tanto el orden natural como el orden social (Jasanoff, 2004). Así la OMS define, siguiendo la terminología de Barnett y Finnemore, tareas compartidas internacionalmente (como "salud"), crea y define nuevas categorías de actores, crea nuevos intereses para los actores (como "pro- moción de la salud") y sirve para transferir modelos de organización sanitaria de unas partes del mundo a otras. Sin embargo, pese a su importancia como una de las grandes agencias de las Naciones Unidas, que reúne hoy prácticamente a todos los países del mundo, son pocos los abordajes históricos que se le han dedicado y menos aún los que plantean la vertiente regional europea de la misma. Una reciente excepción es el proyecto de Steve Sturdy, Richard Freeman y Jennifer Smith (2013) sobre salud mental en la región europea de la OMS que matiza la dominante visión tecnocrática al plantear, desde un estudio empírico de caso, la importancia de la autonomía de los interlocutores frente al dirigismo tecnocrático de la OMS y concluyen, sin pretender universalizar sus hallazgos, que es necesario observar el modo concreto como se forma y trabaja la particular comunidad epistémica que proporciona, en cada caso, los nuevos conocimientos.

La historia de la relaciones internacionales de España en el periodo contemporáneo cuenta ya con una estimable tradición que arranca de la década de los años 1960, cuando se amplió el horizonte desde una historia diplomática enaltecedora hasta los enfoques de un internacionalismo historiográfico con una estrategia intelectual más abierta al exterior (Quintana Navarro, 1996). Precisamente uno de los periodos que han concitado un mayor grado de interés es el correspondiente a la dictadura franquista (Pereira Castañares, Cervantes Conejo, 1990; Tusell, 1993; Sánchez González, 2015) ${ }^{1}$, si bien en ningún caso se ha contemplado el estudio o análisis de la implicación de España en la OMS, salvo en la muy reciente aportación de Rosa Ballester (2016).

Por esta razón, dentro de un equipo con miembros de las Universidades de Granada, Castilla-La Mancha, Alicante y Valencia hemos planteado el abordaje sistemático de la internacionalización de la sanidad española en la segunda mitad del siglo XX y la participación española en las comunidades epistémicas generadoras de conocimiento en el entorno de dicho organismo internacional ${ }^{2}$. Empleando los resultados parciales que se van dando a conocer de este proyecto me propongo ofrecer una valoración provisional del primer periodo de colaboración entre España y la OMS.

José Alberto Palanca fue el Director General de Sanidad del periodo mas duro del franquismo (19361957), quien lidió con la excepcionalidad epidémica de posguerra y quien estructuró administrativamente 
la sanidad pública en España a partir de la Ley de Bases de Sanidad Nacional de 1944 en medio de las estrecheces económicas de la autarquía. Fue también quien dirigió el acceso a la Organización Mundial de la Salud y el establecimiento de protocolos de colaboración con la misma. En lo que sigue, luego de una muy sucinta biografía del personaje, pasaré a contestar las siguientes preguntas: ¿Qué buscaba España al ingresar en la OMS? ¿Cómo se desarrollaron las relaciones entre la OMS y España?¿Qué efecto tuvieron sobre la dinámica profesional y científica de la medicina española?

\section{PALANCA, MILITAR, CONSERVADOR Y SANITARIO}

La guerra había acabado con una Jefatura de Sanidad civil en el bando vencedor, que sería renombrada como Dirección General al finalizar la contienda y que estaba ocupada por un militar médico de carrera, José Alberto Palanca Martínez-Fortún (1888-1973). Licenciado por la Facultad de Medicina de Granada, hizo carrera militar en el Ejército de África, ingresó en 1921 en el cuerpo de inspectores provinciales de Sanidad y, ya comandante médico, entró en la Universidad como catedrático de Higiene de Sevilla en 1923. De Sevilla fue trasladado a Madrid como Inspector provincial, mientras seguía ocupando puestos de responsabilidad en Sanidad militar, merced a un oportuno cambio en la legislación que permitió la movilidad forzosa de dichos puestos, por voluntad ministerial, en 1926 (Molero Mesa y Jiménez Lucena, 2000). Después de una estancia de seis meses en Estados Unidos e Italia, becado por la Fundación Rockefeller en razón a su peso político, como amigo del Dictador y futurible Director general de Sanidad pese a que en opinión del representante de la Fundación "no sabía prácticamente nada de salud pública moderna" 3- llegó efectivamente a ocupar dicho puesto en el Gabinete Berenguer. Pero no mejoró un ápice su valoración ante la Rockefeller: "Bajo ninguna circunstancia [consideraríamos] financiar nada que estuviera dirigido por Palanca, porque sería tirar el dinero" ${ }^{4}$. No está de más recordar que el siguiente enviado de la misma Fundación alabó como "centrista" a Palanca dentro del gobierno de Franco y negoció con él a partir de 1939 la reanudación de los acuerdos a través de sendos programas de colaboración sobre tifus y sobre alimentación (Rodríguez-Ocaña, 2014). Abandonó el ejército en 1931, y fue diputado por
Jaén por el Partido Agrario (1933) sin salir elegido en las elecciones de febrero de 1936 (Álvarez Rey, 2009). Al producirse el alzamiento militar, Palanca se puso a las órdenes del General Mola, actuando como Jefe de Sanidad y Director General de Sanidad hasta julio de 1957 y presidente casi vitalicio de la Real Academia Nacional de Medicina (1953-1970: falleció en 1973). En el esquema administrativo franquista, su presencia simbolizó el control por el ala militar-católica de la parcela Sanidad, enfrentada a la falangista que dirigió Trabajo y Previsión. La confrontación fue notoria en cuestiones como el frustrado seguro contra la tuberculosis (Molero, 2001) y la gestión de la medicina del trabajo ${ }^{5}$ y soterrada en cuanto concierne al seguro de enfermedad, mientras mantuvo su puesto político (Marset, Sáez y Martínez Navarro, 1995, p. 237). Su sustitución conllevó la expresión pública del desbarajuste sanitario que vivía España a partir de la creación del Seguro Obligatorio de Enfermedad, si bien su figura resulta característica del político franquista. En palabras de Camilo Alonso Vega, el Ministro de la Gobernación que lo mandó sustituir, se distinguió por su "buena mano derecha y mejor mano izquierda... [atento a resolver rápidamente todos los problemas que] si no eran legales, eran morales, y si no tenían solución, se buscaba el aplazamiento adecuado"6.

\section{LOS OBJETIVOS DE LA ESPAÑA DE FRANCO DE CARA A LA ORGANIZACIÓN MUNDIAL DE LA SALUD}

Como ha explicado Rosa Ballester (2016), en línea con la historiografía contemporánea, el ingreso en la OMS era un paso necesario en la estrategia política del franquismo para conseguir superar su exclusión del concierto internacional acaecida con la derrota alemana en la II GM. Pero fue la situación política internacional, subrayada por la Guerra de Corea (1950-1952), la que determinó la admisión de España en el sistema de Naciones Unidas, lo que no se produjo en un solo acto (Sánchez González, 2015; Rodríguez Martín, 2009). La Declaración política del Departamento de Estado sobre España de 26 de julio de 1948 , donde se proclamó la voluntad de EEUU de incluir paulatinamente a España dentro del concierto económico y militar de los países occidentales abrió el camino ${ }^{7}$. Luego que la Asamblea General en 4 de noviembre de 1950 acordara la retirada de las sanciones dispuestas desde 1946 y recomendara a sus diferentes ramas que aceptaran la entrada de España, 
comenzó un proceso de adscripción que duró hasta diciembre de 1955, cuando ingresó de pleno derecho en la ONU. Así, la entrada en UNESCO ocurrió en diciembre de 1952 y en UNICEF en febrero de 1954 (Bosch-Marín y Blanco-Otero, 1955). La OMS solicitó la unión de España, que se culminó el 28 de mayo de 1951 con la ratificación por el Estado español de la Constitución de aquella, lo que significó su ingreso como 770 estado miembro en el organismo internacional $^{8}$ (OMS, 1951, p. 155). Como explicó Palanca en el curso de la IV Asamblea Mundial de la Salud que aprobó su ingreso, España había mantenido una actitud de simpatía hacia la nueva Organización e incluso de cooperación en materia de información desde sus inicios (Ballester Añón, 2016, p. 26). Este movimiento coincidió con la retirada de la OMS de los países del bloque socialista soviético (1949-1950), que no se resolvería hasta 1955-1957, por lo que la admisión de España no dejaba de ser un movimiento de peones en el gran tablero de ajedrez de la Guerra Fría (Rodríguez-Ocaña y Porras Gallo, 2017).

Muestra del significado político de dicho proceso la encontramos al analizar las intervenciones de $\mathrm{Pa}$ lanca ante las sucesivas Asambleas Mundiales de la Salud, el plenario legislativo de la OMS, o las de Gerardo Clavero ante el Comité regional europeo: prácticamente en su totalidad, cuando no se trataba de fórmulas de cortesía, eran mociones, reclamaciones o propuestas en relación con la situación colonial, en particular sobre el Protectorado español del norte de Marruecos. Por ejemplo, en la Segunda sesión del Comité regional (Lisboa, 1952) se reclamó contra la posible concesión de estatus de miembro a la zona internacional de Tánger, por ser perteneciente al territorio amparado por España. Y ante la VII Asamblea (1954) al hablarse sobre Marruecos, la delegación española insistió siempre en que se incluyera la mención entre paréntesis "protectorado francés" o español, en su caso, oponiéndose a que se empleara el nombre del país sin su subrayado colonial ${ }^{9}$. Hemos de considerar que, al establecerse la distribución regional dentro de la OMS, Francia colocó a su colina marroquí en Europa, mientras que España decidió que su protectorado se ubicara en la región africana, ambos bajo el paraguas ad hoc para las colonias de "miembros asociados". La única intervención del delegado español en los comités regionales europeos después de la de 1952 fue en 1954, para proponer Madrid como sede de la reunión de 1956, cuando ya se habían presentado con la antelación y formalidad debidas las de Marruecos -zona francesa y Protecto- rado español. En 1956, precisamente, coincidió la muerte del primer director regional, Norman Begg ${ }^{10}$ con la admisión de Marruecos, recién independizado, como miembro de pleno derecho de la Región Europea y la Comisión regional se reunió en Ginebra sin presencia española por primera vez desde su ingreso.

Como imperativo de la propia idiosincrasia del Régimen que exaltaba la España Grande ("una, grande y libre" era la divisa de la España franquista), era preciso situar a España en buena posición en la carrera por el progreso tecnológico y científico mientras que de cara al interior "las tecnologías médicas [podríamos decir la medicina a secas] se convirtieron en uno de los recursos esenciales para vincular el régimen a los ideales de modernización y progreso" (Menéndez Navarro 2007, p. 392). La necesidad del intercambio científico como vía de progreso en el campo médico había quedado establecida en nuestro país a lo largo de los primeros decenios del siglo XX gracias a la labor de la JAE, por lo que se refiere en particular al fomento de la investigación básica en las universidades, sustituida a partir de 1940 por un nuevo Consejo Superior de Investigaciones Científicas, mientras que en el terreno aplicado lo fue mas la actividad de la Comisión de Investigaciones Sanitarias republicana (CIS) y la voluntad de científicos como Gustavo Pittaluga (1876-1956), de quien hay que recordar, junto con su altura intelectual y su activo currículo como gestor de organizaciones sanitarias y periodismo científico, su papel de hombre-puente con la Junta de Sanidad Internacional (IHB) de la Fundación Rockefeller y la Organización de Higiene de la Sociedad de Naciones (Rodríguez-Ocaña, 2010 y 2013). La situación de España en el entramado científico mundial se había deteriorado sustancialmente tras las dos guerras, la interior y la mundial; a partir de 1942, una vez que abandonó España la Fundación Rockefeller a finales del año anterior, no tenía otros interlocutores que los que conformaban "el internacionalismo del Eje" (Brydan, 2016), si bien durante la segunda mitad de la década de 1940 las conveniencias británica y norteamericana mantuvieron vías de comunicación a través de actuaciones de la propia Fundación Rockefeller -cuyo director visitó España en febrero de $1946^{11}$ - o el British Council. A partir del enunciado de la doctrina Truman que oficializó la guerra fría, estas se aceleraron y se acompañaron de la reinstauración de un sistema de becas al exterior por parte del Ministerio de Asuntos Exteriores. Recuperar una situación de normalidad en los intercam- 
bios científico-técnicos con el mundo occidental fue, pues, otro de los objetivos implícitos en la entrada en la OMS. Los acuerdos de Ayuda Económica y Defensa Mutua firmados con EEUU en 1953 no sólo garantizaban la permanencia del régimen franquista, sino que incluían un apartado de colaboración en materia científica y técnica.

Por otra parte, de cara al exterior se buscaba contribuir a la construcción de lo que ahora se llama "marca España", esto es ayudar a difundir una determinada imagen agradable de la España de Franco: como país pacífico, acogedor, culto, científico, moderno, imagen que contrarrestara la carga negativa que a los ojos del público de las democracias liberales guardaba un gobierno dictatorial. En esa línea hay que incluir la promoción de la celebración de eventos científicos internacionales, seminarios y congresos, en España, acometida con fruición por el franquismo. Una revisión catalográfica en REBIUN, completada con algunos hallazgos en otros fondos bibliotecarios pone de manifiesto que, entre 1945 y 1949, se celebró sólo una reunión internacional, hispano-portuguesa, de tema médico, número que ascendió a nueve entre 1950 y 1954, y a doce en el quinquenio siguiente $^{12}$. En conjunto, los Congresos de tema médico o veterinario supusieron el $16 \%(1 / 6), 47 \%$ (9/19) y $44 \%(12 / 27)$, respectivamente, de los celebrados en los tres quinquenios indicados y desde luego fueron la enorme mayoría de los de tipo científico.

Pues bien, el despegue tecnocientífico era impensable sin conexiones internacionales, sin que España se integrara en las redes de producción e intercambio de conocimientos, instrumentos, normas y personas que resumía la existencia de la OMS.

\section{¿CÓMO SE DESARROLLARON LAS RELACIONES ENTRE LA OMS Y ESPAÑA?}

Es habitual dividir en dos tipos las actuaciones emprendidas por la Organización sanitaria mundial para cumplir sus objetivos, acciones normativas y cooperación técnica. Las primeras tienen ámbito regional o universal mientras que las segundas implican a gobiernos o países concretos (Lee y Fang, 2013, p. 9) ${ }^{13}$. La dimensión normativa incluye el estímulo a la formación especializada, mediante cursos en centros de excelencia, y la promoción de la cooperación interprofesional internacional, favoreciendo la celebración de congresos, coloquios y viajes de estudio, para los que se dotan becas, así como reuniones de expertos bajo diversos formatos cuyas conclusiones sirven de vademécum doctrinal. Las intervenciones técnicas exigen la petición de los gobiernos y se concretan en proyectos específicos. En lenguaje OMS "proyecto" era la unidad técnica administrativa de cierta prestación de servicios establecida a petición de un gobierno, compuesta de cuatro momentos estructurantes: la encuesta inicial, el examen epidemiológico, la ejecución y el análisis final (OMS, 1958, p. 170).

El primer paso consistía en el establecimiento de un Acuerdo de Cooperación, que recogía las responsabilidades de cada parte en el contrato y cuyo texto era sustancialmente el mismo para todos los países ${ }^{14}$. Conversaciones, que todavía no conocemos en detalle, si es que conseguimos conocerlas alguna vez, celebradas a lo largo de 1951 entre la directiva regional de la OMS y el gobierno español, produjeron un Acuerdo de Bases de Asistencia Técnica, firmado en Madrid y Ginebra a finales de enero de 1952 (Ballester Añón, 2016) ${ }^{15}$. A continuación se establecían acuerdos supletorios mediante la aprobación consensuada de sucesivos Planes de operaciones, donde se detallaban las fases y contenidos del proyecto concreto. Los dos primeros acuerdos supletorios fueron sobre enfermedades endemo-epidémicas, en particular, leptospirosis (Rodríguez-Ocaña 2017), brucelosis (González Hernández et al., 2018) y rabia (Báguena Cervellera y Mariño Gutiérrez, 2017) y sobre venéreas, en relación con la salud materno-infantil (Castejón Bolea y Rodríguez-Ocaña, 2017). Pero antes de la existencia de acuerdo formal alguno, España ingresó en el Programa de vigilancia mundial sobre Gripe, asignándose al laboratorio de virus de la Escuela Nacional de Sanidad el carácter de Centro Nacional asociado a dicho Programa (Ramírez Ortega y Porras Gallo, 2014).

La Oficina Regional europea de la OMS encargaba a un experto o grupo de expertos, de su plantilla o consultores ad hoc, el asesoramiento a las autoridades locales, siempre decisivas, con actuaciones que incluían demostraciones técnicas o de laboratorio, clínicas y sobre el terreno, la celebración de reuniones con expertos nacionales y la realización de cursos. Su finalidad general era fortalecer los servicios sanitarios nacionales (OMS, 1958, pp. 150-152). Los recursos materiales -financiación, mano de obra, locales - corrían a cargo de los gobiernos, si bien los de tipo más técnico eran completados por otra agencia asociada dentro del sistema de Naciones Unidas, como UNICEF, 
suministradora de instalaciones de rayos $X$, incubadoras, suministros para ortopedia y similares ${ }^{16}$.

En general la existencia de un Proyecto implicaba alguna ausencia, teórica o material, en el funcionamiento diario de los servicios nacionales, que era reconocida como problemática por ambas partes, la local y la instancia internacional. Dicha ausencia era definida en términos normalizados por los cuadros y comités de expertos encuadrados dentro de la OMS, suministradores de una dirección técnica, que era la que presidía la transferencia científico-tecnológica. Por ello, la solicitud de ayuda representaba un arma de dos filos de cara a la imagen del gobierno, lo que nos lleva a pensar que sólo se realizaba cuando en el interior de la administración sanitaria existía una tendencia significativamente favorable; es decir, que existía alguna personalidad, grupo o institución influyente que viera su posición favorecida con y tras las aportaciones internacionales. Los consultores de la OMS enviaban periódicamente informes de la actividad en ejecución, tanto a los gobiernos locales como a su Organización, y estos constituyen las fuentes más importantes de que gozamos para su estudio, cuando disponemos de ellos. El gobierno español, en tiempos de Palanca, no hizo público ninguno.

Las conversaciones para el establecimiento del acuerdo general sin duda incluyeron detalles y especificaciones sobre las áreas consideradas problemáticas. Entre 1951 y 1952 se produjeron las visitas de al menos cuatro enviados de la OMS, el director regional interino y tres consultores, un paludólogo, un experto en rabia y otro en venéreas, acompañados con el envío de un "lote importante" de fumigadoras para emplear con insecticida $\mathrm{HCH}$, alternativa nacional al DDT, de procedencia igualmente suiza (RodríguezOcaña, Perdiguero, y Ballester, 2003), envío de bibliografía actualizada a la Escuela Nacional de Sanidad y asignación de cuatro becas (OMS, 1952, p. 35 y 117). En realidad, el proceso de negociación consistía en un acercamiento entre las partes para detectar los nodos significativos en el sistema, que eran las personas clave, capaces de garantizar el cumplimiento de los acuerdos, o al menos generar confianza en que harían lo posible por hacerlo. La importancia de los vínculos personales y complicidades amistosas con personas significadas de la administración sanitaria del país en cuestión es algo que se observa en la correspondencia del primer decenio de actividades de la OMS, pero que no he encontrado en la que se refiere a España, salvo en referencias a la exquisita cortesía y amabilidad de la recepción. Si se advierte, en cambio, la escasa coordinación que mostró la parte española, donde los diplomáticos del servicio exterior y los sanitarios de la Dirección General de Sanidad no parecían tener una comunicación fluida. De hecho, tanto en 1955 como en 1957, al poco de la sustitución de Palanca, personalidades responsables dentro de Exteriores solicitaron al cuartel general de la OMS una copia de todos los documentos enviados a España ${ }^{17}$.

Como proyectos concretos, en tiempos de Palanca se iniciaron un total de cinco, dedicados, respectivamente, a enfermedades endemo-epidémicas en 1952 (E1 y E2) ${ }^{18}$, higiene materno-infantil (E10) en 1954 (Ballester, 2016) $^{19}$, enfermedades venéreas (E8) (Castejón Bolea y Rodríguez-Ocaña, 2017) y tracoma (E11) en 1955 y rehabilitación de discapacitados infantiles (E3) en 1956 (Ballester, 2016). Es significativa esta relación, tanto por lo que dice -la estrategia pronatalista parece ser la que rige- como por lo que falta en ella, pues se dejan fuera algunos de los mas importantes problemas de salud, por ejemplo el paludismo (bien que es cierto que se concedió alguna beca para viajes al extranjero y cursos) y la tuberculosis. Esta última fue descartada tras la visita del consultor Hood en 1952; el jefe de la sección correspondiente, J. McDougall ${ }^{20}$, indicó en su momento que lo hacían por consenso entre las partes, no porque no fuera necesario sino a cambio de intervenir en zonas mas urgentes. Adviértase que tanto la lucha antipalúdica como la antituberculosa era ámbitos sensibles dentro de la Sanidad española del momento: una estaba personalmente encargada a Gerardo Clavero del Campo, el Director de la Escuela Nacional de Sanidad y máximo responsable de la investigación en salud pública, mientras que la otra, regida por su propio Patronato, gozaba, por su dimensión y presupuesto, de su propia autonomía.

En el curso de su realización se producirían reiteradas visitas de consultores a España, el suministro de ciertos materiales y la dotación de becas para formación, asistencia a cursos, conferencias y coloquios. Entre 1952 y 1955 España recibió 118 becas de las diversas modalidades, de las 2.053 concedidas, algo menos de un $6 \%$ del total ${ }^{21}$. De forma paralela, se incorporó España al circuito de consultas de cara a las conferencias regionales (por ejemplo, en 1952 dos expertos en enfermería y educación sanitaria, respectivamente, vinieron para preparar próximas reuniones europeas) (OMS, 1953, p. 121), como tam- 
bién lo hizo, paulatinamente, al circuito formativo, de tal manera que centros nacionales recibirían cierto número de becarios OMS procedentes de terceros países a partir de 1955 (un becario italiano para estudiar la población de anófeles en Valencia, en 1955; sendos becarios de los territorios belgas y portugueses africanos para estudiar lepra; otro portugués para asistir a curso de venereología, en 1956; un becario de Marruecos en administración sanitaria y otro portugués para estadística sanitaria en 1957) y entró a formar parte de las sedes elegibles para la celebración de conferencias y coloquios: lo más significativo en este terreno fue la celebración del Seminario - la prensa lo denominó "Congreso"- sobre "Aspectos de las enfermedades producidas por virus y por rickettsias que interesan a los laboratorios de Salud Pública" que se reunió en Madrid en abril de 1956 a lo largo de diez días con participación de profesionales de 35 países $^{22}$. Por último, la OMS incorporó a un número de profesionales españoles a sus listas de expertos; entre los primeros estuvieron Eugenio Sellés Martí (1904-1997), Benigno Lorenzo Velázquez (1901-1985), José Gay Prieto (1905-1979), todos ellos catedráticos en Madrid a partir de 1940, o Gerardo Clavero del Campo (1895-1972), el director de la Escuela Nacional de Sanidad desde 1941, incorporando las funciones del Instituto homónimo que existía con anterioridad, como institución central para la investigación sanitaria.

\section{EFECTOS SOBRE LA DINÁMICA PROFESIONAL Y CIENTÍFICA DE LA MEDICINA ESPAÑOLA}

Estos apenas seis primeros años de relaciones con la OMS tuvieron un efecto limitado y localizado en las dinámicas profesionales y científicas sanitarias, porque se plantearon desde una perspectiva sanitarista muy concreta y por su extremado centralismo. En efecto, se trató sobre todo de reforzar la vertiente de laboratorio a través de dispositivos oficiales preexistentes, casos de la lucha antivenérea o la Escuela Nacional de Sanidad, donde ese fortalecimiento se logró andando el tiempo, con destacada participación y liderazgo de Florencio Pérez Gallardo (1917-2006) (Báguena Cervellera, y Mariño Gutiérrez, 2017; Porras Gallo y Ramírez, 2017; Báguena Cervellera y Mariño, 2016; Báguena, Porras y Caballero, 2014; Ramírez Ortega y Porras Gallo, 2014). En el terreno de la rehabilitación, la influencia exterior resultó un acicate fundamental para la implantación de alguna especia- lidad. Por otro lado, hay que consignar que sus primeros y principales beneficiarios fueron grupos, personas e instalaciones situadas en Madrid.

Se consiguió el propósito de superar el aislamiento internacional, en el plano formal, con la entrada en la OMS, si bien España se incorporó con lentitud a la norma europea en cuanto a promotora y sede de reuniones internacionales. Las becas fueron concedidas en su mayoría a personas de los cuerpos de Sanidad Nacional, por lo que tendrían valor igualmente como premio al desempeño habitual. Esa condición de premio parece tener, igualmente, el acudir anualmente a la reunión del Consejo regional (Regional Committee, RC) europeo, pues en todos esos años no repitió nadie (Clavero RC2, Pastor Krauel RC3, Manzanares RC4, Pérez Gallardo RC5, nadie en RC6). Se hizo posible la circulación de las publicaciones de la OMS, como prueban las colecciones de la serie de Informes técnicos que se localizan en las bibliotecas universitarias españolas (Madrid, Barcelona, Valencia, etc.), lo que permitió un acceso a información doctrinal actualizada. El proyecto de lucha contra la sífilis congénita garantizó el funcionamiento de la escuela de especialidad en Dermatología y Venereología vinculada a la Facultad de Medicina de Madrid, creada sobre el papel en 1945. Los viajes a Europa, los cursos y la consulta regular de publicaciones fueron elementos necesarios para garantizar la incorporación española a los circuitos internacionales de comunicación y transferencia científicas, que no sólo se produjeron en el contexto de la vinculación con la OMS, sino también en el de los Acuerdos hispano-norteamericanos y en la gestión personal de muchos investigadores ambiciosos en el terreno profesional, con o sin aval oficial.

El general Palanca, africanista, vino a ser destituido de la DGS justo después de la independencia de Marruecos y en los inicios de la guerra de Ifni. Simbolizaba otra época. Su mano derecha en materias de investigación, Clavero, sin embargo, le sobrevivió en el puesto hasta 1965. Por lo que se refiere a las contribuciones españolas, no hay constancia de que nuestras más altas autoridades sanitarias desempeñaran rol alguno en el diseño o modificación de recomendaciones y políticas sanitarias de la OMS. No se les recuerda ninguna intervención en tal sentido. En cambio, existieron algunas contribuciones expertas, notablemente eficaces, las de Gay Prieto y Pérez Gallardo, que suscitaron reconocimiento: por ejemplo, cuando Gay Prieto abandonó, por jubilación, el 
panel de expertos en treponematosis, fue incorporado como consultor por el secretariado de la OMS.

Las relaciones con la OMS se reforzaron notablemente en las dos décadas siguientes. En ellas, los mecanismos de comunicación iniciados durante este primer sexenio se perfeccionaron y ampliaron y varios de los proyectos iniciados alcanzaron sus mejores frutos, que confiamos seguir estudiando en próximos trabajos.

\section{NOTAS}

1 También Del aislamiento a la apertura: La política exterior de España durante el franquismo, (2006), III Jornadas de la Comisión Española de Historia de las Relaciones Internacionales, Universidad de Burgos, 2004, Madrid: Comisión Española de Historia de las Relaciones Internacionales.

2 Proyectos HAR2015-70688-C2-1-P (MINECO/FEDER) y HAR2015-70688-C2-2-P, MINECO y Fondos FEDER.

3 Carta de Bailey a Russell, 12-12-1926, Archivos de la Fundación Rockefeller, RF, 5, 1.2, 273, 3463.

4 Memorandum Dr. Pascua, (RBH), 26 de octubre de 1934. Archivos de la Fundación Rockefeller, RF, RG 6.1, 1.1, 34, 422.

5 Rodríguez-Ocaña, Esteban y Ortiz, Teresa (2008), Medical Research in Franco's Spain. An Overview. Ponencia presentada al Simposio "Science and totalitarian systems", Barcelona 20-22 Nov 2008.

6 Toma posesión el Director General de Sanidad. "Se impone una urgente colaboración de los servicios sanitarios", dijo el ministro de la Gobernación. ABC, 23 julio 1957 (edición matutina), p. 2.

7 Department of State. United States of America. Policy Statement by the Department of State on Spain, July 26, 1948. Doc 656. Office of the Historian, history.state.gov [consultado el 8 de noviembre de 2017].

8 OMS (1951), p. 155

9 Archivo de la OMS, Ginebra. EUR/RC2/Min 3 [Minutas del 20 Comité Regional Europeo, punto tercero], p. 15; EUR/ RC2/Min 5, p. 4; WAH7_VR-9 [7a Asamblea Mundial de la Salud, Novena reunión plenaria, actas -verbatim records-] y WHA7_VR-9Corr.1 [Actas de la 7a Asamblea Mundial de la Salud, Novena reunión plenaria, corrigendum 1].

10 Norman Darroch Begg (1906-1956), médico escocés y doctor por la Universidad de Aberdeen, había sido el delegado de UNRRA en Polonia en 1946 y formó parte de la Comisión Interina de la OMS.

11 Hay una foto de esta visita en Toledo publicada en Notas gráficas de actualidad, ABC, 27 de febrero de 1946, p. 5.

12 El VI Congreso Internacional de Patología Comparada (1952), el VI Congreso Internacional de Leprologia (1953), o el IV Congresos internacional de de Medicina Interna (1956) se reunieron en Madrid y el IV Congreso de Higiene

\section{AGRADECIMIENTOS}

Trabajo realizado en el marco del Proyecto HAR2015-70688-C2-1-P (MINECO-FEDER). Una versión preliminar de este trabajo se presentó en la Mesa redonda "La apertura internacional del régimen franquista: ¿obligación u oportunidad para la modernización del país?" celebrada en Ciudad Real el 15 de noviembre de 2017.

y Medicina Mediterráneas (1953), el III Congreso Internacional de las enfermedades del tórax, del American College of Chest Physicians y el VI (1958) de Medicina Interna, tuvieron lugar en Barcelona.

13 Citan a Fluss y Gutteridge, 1993

14 A partir de 1954 se modificó dicho texto, debido a que se habían suscitado problemas en ciertos lugares, sobre responsabilidad económica de la OMS respecto a sus misiones locales, por lo que hubo que volver a firmarlo. En una lista de 1958, sin fecha, España aparecía pendiente de cambiar el tratado junto con otros quince países de todo el mundo y distintas circunstancias socioeconómicas. Archivo de la OMS, Ginebra, 1955-1961 Technical Assistance Basic and Supplementary Agreements, WHO.2, $\mathrm{T} 2 / 36 / 2$.

15 No hemos localizado mas que documentación de tipo administrativo sobre esta firma (correspondencia solicitando direcciones, etc.) así como una copia del texto en: Archivo de la Organización Mundial de la Salud, Ginebra. Basic agreement concerning technical asístanse, WHO2$\mathrm{PH}-2 / 5 / 50$. El fichero SPAIN UNDP Technical assistance to Spain, WHO 3 4, T2/372/4 del mismo Archivo, debería contener la documentación correspondiente a las directrices y negociaciones llevadas a cabo entre 1955 y 1962, pero consta explícitamente que fue destruida.

16 Ballester, Rosa y Rodríguez-Ocaña, Esteban, «Joint activities WHO-UNICEF in the field of Child Health Services (19491962): a case study", European Social Sciences and History Conference, Belfast, 4-7 April 2018.

17 Archivo de la OMS, Ginebra. 1955-1992 Non technical relations with the government of Spain, WHO3rd_4 $4^{\text {th }}$, N52-180-2SPA.

18 Archivo de la OMS, Ginebra. SPA-2 Supplementary Agreement No. 1 to the Basic Agreement between the World Health Organization and the Government of Spain covering Zoonoses, including Q Fever, Brucellosis and Rabies signed at Madrid on 24 January and Geneva on 30 January 1952. WHO2_PH 2/5/50. González Hernández et al. (2018).

19 Ballester, Rosa y Rodríguez-Ocaña, Esteban, «"Nacidos demasiado pronto". La prematuridad en la agenda de la Salud Materno-Infantil de la OMS y el Plan de Operaciones para España (1954-1964)», Simposio "Salud, Enfermedad y Fran- 
quismo", Ciudad Real, 27-28 de junio de 2018 (en prensa).

20 El médico escocés, y jugador internacional de rugby, John Bowes MacDougall (1890-1967) tuvo un papel destacado en la introducción de la tomografía para el examen radiológico de la tuberculosis pulmonar.

21 Archivo de la OMS, Ginebra. Report of the regional director WHO fellowship programme in Europe. General review

\section{BIBLIOGRAFÍA}

Álvarez Rey, Leandro (2009), Los Diputados por Andalucía de la Segunda República 1931-1939, Sevilla, Fundación Centro de Estudios Andaluces.

Báguena Cervellera, María José; Mariño Gutiérrez, Lourdes (2016), "Economía y salud: costes y beneficios de la erradicación de la viruela en España mediante la vacunación (1959-1982)". En: Porras Gallo, Maria Isabel; Báguena Cervellera, María José; Ayarzagüena Sanz, Mariano; MartínEspinosa Noelia María (coords.), La erradicación de las enfermedades infecciosas: viruela y poliomielitis, Madrid, La Catarata, pp. 149-169.

Báguena Cervellera, María José; Mariño Gutiérrez, Lourdes (2017), "La participación española en los programas de la OMS para el control de la rabia (1952-1975)". En: Zarzoso, Alfons; Arrizabalaga, Jon (eds.), Al servicio de la salud humana: La historia de la medicina ante los retos del siglo $X X I$, Sant Feliu de Guíxols, SEHM, Museu d'Història de la Medicina de Catalunya, Museu d'Història de Sant Feliu de Guíxols, Institució Milà i Fontanals, pp. 117-122.

Báguena, María José; Porras Gallo, María Isabel; Caballero, María Victoria (2014), "Innovación, producción y circulación de vacunas contra la viruela y la polio en España (19181963)". En Campos Marín, Ricardo; González De Pablo, Ángel; Porras Gallo, María Isabel; Montiel, Luis (eds.), Medicina y poder político. XVI Congreso Sociedad Española de Historia de la Medicina Madrid, SEHM-UCM, pp. 197-202.

Ballester Añón, Rosa (2016), España y la Organización Mundial de la Salud en el contexto de la historia de la salud pública internacional (1948-1975). Discurso de recepción ..., Valencia, Real Academia de Medicina de la Comunidad Valenciana, pp. 3-78.

Barnett, Michael N; Finnemore, Martha (1999), "The politics, power, and pathologies of international organizations," International Organization, 53 (4), pp. 699-732.

Barona, Josep Lluís (2015), The Rockefeller Foundation, Public Health and International Diplomacy, 1920-1945, New York, Routledge.

Birn, Anne-Emanuelle, (2006), Marriage of convenience: Rockefeller International Health and revolutionary Mexico, Rochester, NY, University of Rochester Press.

Birn, Anne-Emanuelle, (2014a) "Backstage: the relationship between the Rockefeller Foundation and the World Health covering the eight year period 1947-1954, EUR/RC5/2 Add.1 [5o Comité Regional Europeo, punto 2, anexo 1]; Draft programme and budget estimates (para 1956, 1957 y 1958), en EUR/RC6 [6ㅇ Comité Regional Europeo], EUR/ RC7 [7을 Comité Regional Europeo] y EUR/RC8 [8ㅇ Comité Regional Europeo]

22 Clausura del Congreso de la Organización Mundial de la Salud. ABC, 26 de abril de 1956, p. 27.

Organization, Part I 1940s-1960s", Public Health, 128 (2), pp. $129-140$

Birn, Anne-Emanuelle (2014b), “Philanthrocapitalism, past and present: The Rockefeller Foundation, the Gates Foundation, and the setting(s) of the international/ global health agenda", Hypothesis, 12(1): e8, [en línea] disponible en: http://www. hypothesisjournal.com/wp-content/uploads/2014/11/ HJ229\%E2\%80\%94FIN_Nov1_2014.pdf [consultado el $12 / 05 / 2018]$

Borowy, Iris (2009), Coming to terms with world health: the League of Nations Health Organisation 1921-1946, Frankfurt am Main, Peter Lang .

Borowy, Iris (2014), "Shifting Between Biomedical and Social Medicine: International Health Organizations in the 20th Century", History Compass, 12 (6), pp. 517-530, [en línea] http://dx.doi.org/10.1111/hic3.12162.

Bosch-Marín, Juan y Blanco-Otero, Manuel (1955), UNICEF, Madrid, Mo Gobernación- Dirección General de Sanidad.

Brown, Theodore, Cueto, Marcos (2011), "The World Health Organization and the World of Global Health". En: Richard Parker; Marni Sommer (eds.), Routledge Handbook in Global Public Health, New York, Routledge, pp. 18-30

Brown, Theodore M, Cueto, Marcos y Fee, Elizabeth (2006), "The World Health Organization and the transition From "international" to "global" public health". American Journal of Public Health, 96(1), pp. 62-72.

Brydan, David (2016), "Axis Internationalism: Spanish Health Experts and the Nazi 'New Europe', 1939-1945", Contemporary European History. 25 (Special Issue 02), pp. 291-311 [en línea] http://dx.doi.org/10.1017/S0960777316000084

Castejón Bolea, Ramón; Rodríguez-Ocaña, Esteban (2017), “EI control de las enfermedades venéreas en España durante la década de 1950: los inicios del Proyecto España 8 de la OMS". En: Zarzoso, Alfons; Arrizabalaga, Jon (eds.), Al servicio de la salud humana: la Historia de la Medicina ante los retos del siglo XXI, Museu d'Història de la Medicina de Catalunya, Museu d'Història de Sant Feliu de Guíxols, Institució Milà i Fontanals (CSIC), pp.101-108.

Cueto, Marcos (2004), El valor de la salud: historia de la Organización Panamericana de la Salud. Washington, D.C.: Organización Panamericana de la Salud. 
Cueto, Marcos; Brown, Theodore; Fee, Elisabeth (2011), "El proceso de creación de la Organización Mundial de la Salud y la Guerra Fría", Apuntes. Revista de Ciencias Sociales, 38(69), pp. 129-156.

Farley, John (2004), To Cast Out Disease. A History of the International Health Division of the Rockefeller Foundation (1913-1951), Oxford, Oxford University Press.

González Hernández, María; Ballester Añón, Rosa; Porras Gallo, María Isabel y Báguena Cervellera, María José (2008), “Ayuda técnica en tiempos de crisis: la brucelosis en los programas país para España de la Organización Mundial de la Salud (1951-1972)". Rev. Esp. Salud Pública, Vol. 92; e1-e11 [en línea], disponible en: scielo.isciii.es/pdf/resp/v92/11355727-resp-92-e201810058.pdf [consultado el 15/06/2018].

Haas, Peter M. (1992), “Introduction: Epistemic communities and international policy coordination", International Organization, 46, (1) pp. 1-35.

Hussein, Ali (1998a), "WHO at fifty: 1. Highlights of the early years until 1960", World Health Forum, 19(1), pp. 21-37.

Hussein, Ali (1998b) "WHO at fifty: 2. Highlights of activities from 1961 to 1973", World Health Forum, 19(2) , pp. 140-155.

Iriye, Akira (2002), Global community. The role of international organization in the making of contemporary world, Berkeley, University of California Press.

Jasanoff, Sheila (2004), States of knowledge: The co-production of science and the social order, New York, Routledge.

Lee, Kelley; Fang, Jennifer (2013), Historical dictionary of the World Health Organization, Lanham-Toronto-Plymouth, The Scarecrow Press.

Litsios, Socrates (2009), The third ten years of the World Health Organization, Geneva, WHO.

Litsios, Socrates (2012), The fourth ten years of the World Health Organization, Geneva, WHO.

Löwy, Irina, Zylberman, Patrick, (2000), "Medicine as a Social Instrument. Rockefeller Foundation, 1913-45". Studies in the History and Philosophy of Biological and Biomedical Sciences, 31 (3), pp. 365-3799.

Marset, Pedro; Sáez, José Miguel; Martínez Navarro, Ferrán, (1995), "La Salud Pública durante el franquismo", Dynamis, 15 , pp. $211-250$

Menéndez Navarro, Alfredo (2007), “Átomos para la Paz... y para la Medicina: la popularización de las aplicaciones médicas de la energía nuclear en España". Revista Española de Medicina Nuclear, 26 (6), pp. 385-399.

Molero, Jorge (2001), "Health and public policy during the early Francoist regime (1936-1951): the tuberculosis problem". En: Löwy, Irina; Kriege, John (eds.), Images of disease. Science, public policy and health in post-war Europe, Luxembourg, European Commission; pp. 141-165.

Molero Mesa, Jorge; Jiménez Lucena, Isabel (2000), "Salud y burocracia en España. Los Cuerpos de Sanidad Nacional (1855-1951)", Revista Española de Salud Pública 74, pp. 45-79.
OMS (1951), Activité de l'OMS en 1951. Rapport annuel du Directeur Général à l'Assemblée Mondiale et aux Nations Unies (1952), Actes officiels de l'Organisation Mondiale de la Santé, no 38. Genève, Organisation Mondiale de la Santé.

OMS (1952) Activité de l'OMS en 1952. Rapport annuel du Directeur-Géneral à l'Assemblée Mondiale et aux Nations Unies (1953), Actes officiels de l'Organisation Mondiale de la Santé, no 45. Genève, Organisation Mondiale de la Santé.

OMS (1958), Los Diez Primeros Años, Ginebra, Organización Mundial de la Salud.

OMS (1968), The Second Ten Years of the World Health Organization, 1958-1967, Geneva, World Health Organization.

Pereira Castañares, Juan Carlos; Cervantes Conejo, Ángel (1990), "La política exterior del Franquismo: un reto para la historiografía española", Cuadernos de Historia Contemporánea, 12, pp. 175-182.

Porras Gallo, Maria Isabel; Ramírez Ortega, Mercedes (2017), "La lucha contra la gripe en España a través de las relaciones con la OMS (1951-1971)". En: Zarzoso, Alfons; Arrizabalaga, Jon (eds.), Al servicio de la salud humana: La historia de la medicina ante los retos del siglo XXI. San Feliu de Guíxols, SEHM, Museu d'Història de la Medicina de Catalunya, Museu d'Història de Sant Feliu de Guíxols, Institució Milà i Fontanals, pp. 109-115.

Quintana Navarro, Francisco. (1996), "La historia de las relaciones internacionales en España: apuntes para un balance historiográfico". En: La historia de las relaciones internacionales: una visión desde España: Madrid, 20, 21 y 22 de octubre, Madrid, Comisión española de historia de las relaciones internacionales, pp. 1-65.

Ramírez Ortega, Mercedes; Porras Gallo, María Isabel, (2014), "La vacunación contra la gripe y sus complicaciones en España (1918-2009)". En: Campos Marín, Ricardo.; González De Pablo, Ángel; Porras Gallo, Maria Isabel; Montiel, Luis (eds.), Medicina y poder político. Madrid: SEHM-UCM, pp. 191-195.

Rodríguez Martín, José Antonio (2009), “Integración de España en la organización económica internacional: de la Guerra civil y la autarquía a la Comunidad Económica Europea", Revista de Historia Actual, 7(7), pp. 95-104.

Rodríguez-Ocaña, Esteban (2000), "La intervención de la Fundación Rockefeller en la creación de la sanidad contemporánea en España". Revista Española de Salud Pública, 2000, 74, pp. 27-34.

Rodríguez-Ocaña, Esteban, ed. (2002), The Politics of the Healthy Life: an International Perspective, Sheffield, EAHMH Publications.

Rodríguez-Ocaña, Esteban (2010), "La JAE y la consolidación de la Salud Pública en España". En: Sánchez Ron, José Manuel; García-Velasco, José (eds.), 100 JAE. La Junta para Ampliación de Estudios e Investigaciones Científicas en su centenario, Madrid: Publicaciones de la Residencia de Estudiantes, pp. 601-623. 
Rodríguez-Ocaña, Esteban (2013), “Pittaluga Fattorini, Gustavo". En: Diccionario Biográfico Español, vol. XLI, Madrid, Academia de la Historia, pp. 739-742.

Rodríguez-Ocaña, Esteban (2014), “Por razón de ciencia. La Fundación Rockefeller en España (1930-1941)”. En: Campos Ricardo; González de Pablo, Ángel; Porras Gallo María Isabel; Montiel, Luis (eds), . Medicina y poder político. XVI Congreso Sociedad Española de Historia de la Medicina. Madrid, 11-13 junio 2014. Madrid, SEHM, pp. 473-477

Rodríguez-Ocaña, Esteban (2017), “Ocaso de la medicina social en España: El caso de la leptospirosis”,Asclepio 69 (2): p199. [en línea] http://dx.doi.org/10.3989/asclepio.2017.22

Rodríguez-Ocaña, Esteban; Martínez Navarro, Ferrán (2008), Salud pública en España: de la Edad Media al siglo XXI, Granada, Escuela Andaluza de Salud Pública.

Rodríguez-Ocaña, Esteban; Perdiguero, Enrique; Ballester Añón, Rosa (2003), "La higiene ecologicida. Guerra contra los mosquitos". En: Rodríguez-Ocaña, Esteban; Ballester Añón, Rosa; Perdiguero, Enrique; Medina Doménech, Rosa María; Molero Jorge, La acción médico-social contra el paludismo en la España metropolitana y colonial del siglo XX. Madrid, CSIC, pp. 295-310.

Rodríguez-Ocaña, Esteban; Porras Gallo, María Isabel (2017), "Contactos internacionales de la España de Franco en Medicina y Salud Pública como vía de normalización intelectual y científica. Presentación" En: Zarzoso, Alfons; Arrizabalaga,
Jon (eds. ), Al servicio de la salud humana: la Historia de la Medicina ante los retos del siglo XXI. Sant Feliú de Guixols: SEHM, Museu d'Història de la Medicina de Catalunya, Museu d'Història de Sant Feliu de Guíxols, Institució Milà i Fontanals (CSIC), pp. 89-93.

Roemer, Milton (1993), "Internationalism in Medicine and Public Health", En: Bynum H; Porter Roy (eds.), Companion Encyclopedia of the History of Medicine, London, Routledge; pp. 1417-1435.

Sánchez González, Irene (2015), Diez años de soledad: España, la ONU y la dictadura franquista, 1945-1955, Sevilla: Universidad de Sevilla.

Solomon, Susan Gross; Murard, Lion; Zylberman, Patrick (eds.) (2008), Shifting boundaries of public health: Europe in the twentieth century. Rochester, NY, University of Rochester Press.

Stapleton, Darwin H. (2000), “Internationalism and nationalism: the Rockefeller Foundation, public health, and malaria in Italy, 1923-1951", Parassitologia, 42(1-2), pp. 127-134.

Sturdy, Steve; Freeman, Richard; Smith-Merry, Jennifer (2013), "Making knowledge for international policy: WHO Europe and mental health policy, 1970-2008", Social History of Medicine, 26 (3):, pp. 532-554.

Tusell, Javier (1993), El régimen de Franco. Política y relaciones exteriores, Madrid, UNED. 